\title{
Continuous variable entanglement of counter-propagating twin beams
}

\author{
A. Gatti ${ }^{1,2}$ and E. Brambilla ${ }^{2}$ \\ 1 Istituto di Fotonica e Nanotecnologie del CNR, Piazza Leonardo da Vinci 32, Milano, Italy \\ ${ }^{2}$ Dipartimento di Scienza e Alta Tecnologia dell'Università dell'Insubria, Via Valleggio 11 Como, Italy
}

\begin{abstract}
This work describes the continuous-variable entanglement of the counter-propagating twin beams generated in a Mirrorless Optical Parametric Oscillator below threshold, encompassing both their quadrature and photon-number correlation. In the first case, a comparison with the single-pass co-propagating geometry outlines a completely different stability of the two sources with respect to the phase-angle. In the second case, stimulated by the critical divergence of the correlation time evidenced by Corti et al. , we address the issue of the temporal bandwidth of the intensity squeezing.
\end{abstract}

\section{INTRODUCTION}

Squeezed light and continuous variable entanglement are precious resources for quantum information, communication and metrology. One of the most accessible and widely used source is represented by the twin beams [1] generated through parametric-down conversion (PDC) from a pump laser. This work focuses on a peculiar configuration, where the twin beams are generated in opposite directions, and counter-propagate in a slab of $\chi^{(2)}$ material (Fig. 1). This process is allowed only in the presence of quasi-phase matching in periodically poled crystals, and presents the challenge of requiring very short poling periods [2, 3] on the order of the pump wavelength. Predicted in the sixties [4, counter-propagating PDC had indeed to wait almost forty years before being demonstrated [5].

Counter-propagating PDC emerged in the last years as a promising source of quantum light, with several peculiar and appealing features [6 10. First of all, in contrast with the usual single-pass co-propagating geometry, this source is narrowband, so that in the spontaneous regime it has the potentiality to generate narrowband heralded single photons in almost pure states [6] $[$. The second peculiarity is the presence of a threshold value of the pump intensity,

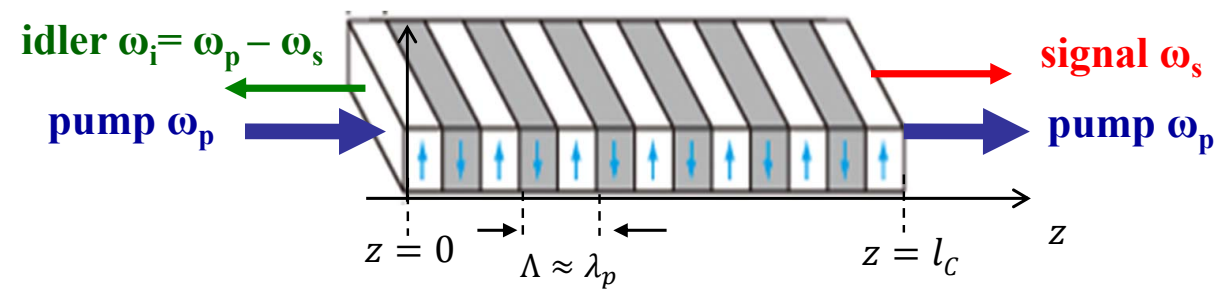

FIG. 1: (Color online) Scheme of counterpropagating parametric down-conversion, taking place in a periodically poled crystal of length $l_{c}$ and poling period $\Lambda \approx \lambda_{p}$. Quasi-phase matching requires that the idler field is generated in the backward direction with respect to the signal and pump.

beyond which the system makes a transition to coherent oscillations, similarly to what happens in a standard Optical Parametric Oscillator (OPO), from which the name Mirrorless Optical parametric Oscillator(MOPO) [5]. Responsible of this critical behaviour is a feedback mechanism, which in this case is established not by the cavity mirrors, but by the back-propagating wave, in combination with stimulated down-conversion [9]. A recent analysis[10] has shown that this cavityless configuration of PDC may produce the same high and stable level of squeezing and quadrature correlation as the OPO, and may thus become a robust and monolithic alternative to the cavity configuration.

This work provides a general description of the continuous-variable entanglement of the MOPO twin beams below threshold, encompassing both their quadrature and photon-number correlation. In the first case, a comparison will be performed with the single-pass co-propagating geometry, which in its high-gain regime can be used as a source of squeezed light 11-13. A part from the huge difference of the bandwidth involved, our analysis will outline a completely different stability of the two sources with respect to the phase-angle at which squeezing takes place. For the photon number correlation, our analysis will address the question of how long the twin beams should be detected in order to observe sub-shot noise fluctuations in the difference of the their photon-numbers, or in other words, the issue of the bandwidth of the intensity squeezing. The interest in this sense is stimulated by the findings of Ref. 99, where it was shown that on approaching the MOPO threshold the twin beams become correlated over a longer and longer time, ideally diverging at threshold.

The work is organized as follow: after briefly introducing the quantum model for the device, and describing the spectral characteristics of the emission (sections II and III), Sec IV reviews some general properties which are 
common to all processes of photon-pair generation. This allows in the next section $\mathrm{V}$ a straightforward analysis of the quadrature correlation in the two counter-propagating and co-propagating configurations. The final Sec VI addresses the problem of the intensity squeezing in the MOPO and of its bandwidth.

\section{THE MODEL}

We consider the geometry in Fig 1 , in which the laser pump and the down-converted signal co-propagate along the $+z$ direction, while the idler back-propagates in the $-z$ direction along a periodically poled slab of a $\chi^{(2)}$ material. Our quantum model for this configuration is described in Refs. [7, 9, 10] (see also [14, [15]). We summarize in the following the main points

- A purely temporal description of the twin beams is carried out, assuming either a waveguided configuration or that a small angular bandwidth is collected.

- The pump laser is described as a monochromatic classical beam of frequency $\omega_{p}$ and amplitude $\alpha_{p}=\sqrt{I_{p}} e^{i \phi_{p}}$. Below the MOPO threshold, we assume that it is undepleted by the parametric interaction, an approximation which clearly becomes unphysical as one gets very close to the MOPO threshold.

- The signal and idler fields are described by quantum field operators for two wavepackets centered around frequencies $\omega_{s}$ and $\omega_{i}=\omega_{p}-\omega_{s}$, such that their corresponding wave numbers in the medium, $k_{j}=\omega_{j} n_{j}\left(\omega_{j}\right) / c$, satisfy the quasiphase matching condition $k_{s}-k_{i}=k_{p}-k_{G}$ where $k_{G}=2 \pi m / \Lambda$ is the reciprocal vector of the nonlinear grating at first or low order, $m=1,3,5$.

- The generation of twin beams in the nonlinear slab is then described by linear parametric equations, that couple the signal and idler field operators via the dimensionless gain parameter

$$
g=\sqrt{2 \pi} \chi\left|\alpha_{p}\right| l_{c}
$$

where $\chi$ is proportional to the $\chi^{(2)}$ nonlinear susceptibility of the medium and $l_{c}$ is the crystal length.

These propagation equations are then solved in terms of linear input-output transformations linking the output field operators to the input ones. Notice that here the boundaries differ from the standard ones, because the output signal and idler fields: $\hat{A}_{s}^{\text {out }}(\Omega)=\hat{A}_{s}\left(\Omega, z=l_{c}\right), \hat{A}_{i}^{\text {out }}(\Omega)=\hat{A}_{i}(\Omega, z=0)$, appear on the opposite faces of the slab (Fig 2 , while the input fields: $\hat{A}_{s}^{\text {in }}(\Omega)=\hat{A}_{s}(\Omega, z=0), \hat{A}_{i}^{\text {in }}(\Omega)=\hat{A}_{i}\left(\Omega, z=l_{c}\right)$, assumed in the vacuum state, enter from opposite faces. The input-otput relations have the general form of a Bogoliubov transformation, characteristic of

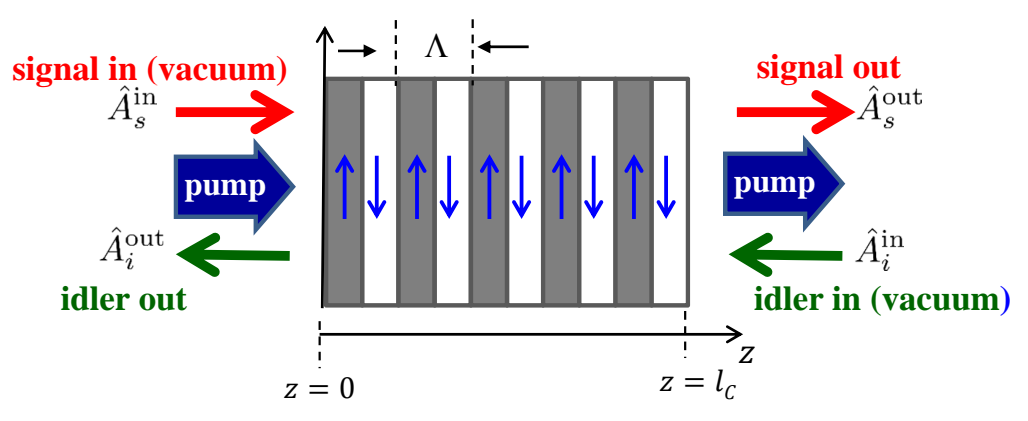

FIG. 2: (Color online) Counterpropagating input-otput scheme.

processes where particles are generated in pairs:

$$
\begin{aligned}
\hat{A}_{s}^{\text {out }}(\Omega) & =U_{s}(\Omega) \hat{A}_{s}^{\text {in }}(\Omega)+V_{s}(\Omega) \hat{A}_{i}^{\text {in } \dagger}(-\Omega) \\
\hat{A}_{i}^{\text {out }}(-\Omega) & =U_{i}(-\Omega) \hat{A}_{i}^{\text {in }}(-\Omega)+V_{i}(-\Omega) \hat{A}_{s}^{\text {in } \dagger}(\Omega) .
\end{aligned}
$$

Here $\hat{A}_{j}^{\text {out,in }}(\Omega) j=, s, i$ are the positive frequency parts of the electric field operators, with dimensions of photon annihilation operators, such that $\hat{A}_{j}^{\text {†out }}(\Omega) \hat{A}_{j}^{\text {out }}(\Omega)$ are the output photon numbers per unit frequency. Capital $\Omega$ denotes the frequency offset from the respective central frequencies $\omega_{s}$ and $\omega_{i}$,

Unlike the co-propagating case, where the coefficients of the input-output transformation grow exponentially with the propagation length in the medium, in the MOPO case the coefficients $U_{j}(\Omega)$ and $V_{j}(\Omega)$ are trigonometric functions 
of the crystal length (through the gain parameter $g \propto l_{c}$ ), and read [9, 14]:

$$
\begin{array}{ll}
U_{s}(\Omega)=e^{i k_{s} l_{c}} e^{i \beta(\Omega)} \phi(\Omega) ; & V_{s}(\Omega)=e^{i\left(k_{s}-k_{i}\right) l_{c}} g e^{i \phi_{p}} \frac{\sin \gamma(\Omega)}{\gamma(\Omega)} \phi(\Omega) ; \\
U_{i}(-\Omega)=e^{i k_{i} l_{c}} e^{i \beta(\Omega)} \phi^{*}(\Omega) ; & V_{i}(-\Omega)=g e^{i \phi_{p}} \frac{\sin \gamma(\Omega)}{\gamma(\Omega)} \phi^{*}(\Omega) .
\end{array}
$$

where:

$$
\begin{aligned}
\phi(\Omega) & =\frac{1}{\cos \gamma(\Omega)-i \frac{\overline{\mathcal{D}}(\Omega) l_{c}}{2 \gamma(\Omega)} \sin \gamma(\Omega)} \\
\gamma(\Omega) & =\sqrt{g^{2}+\frac{\overline{\mathcal{D}}^{2}(\Omega) l_{c}^{2}}{4}}, \\
\mathcal{D}(\Omega) & =k_{s}(\Omega)-k_{i}(-\Omega)-k_{p}+k_{G}, \\
\beta(\Omega) & =\left[k_{s}(\Omega)+k_{i}(-\Omega)-\left(k_{s}+k_{i}\right)\right] \frac{l_{c}}{2}
\end{aligned}
$$

The function $\mathcal{D}(\Omega)$ in Eq. (6) is the phase mismatch of the two frequency conjugate waves at $\omega_{s}+\Omega$ and $\omega_{i}-\Omega$, with $k_{j}(\Omega)=n_{j}(\Omega)\left(\omega_{j}+\Omega\right) / c$ being their wave numbers in the medium. The function $\beta(\Omega)$ in Eq. (7) is instead a global propagation phase.

As can be easily checked, these coefficients satisfy the unitarity conditions

$$
\begin{aligned}
& \left|U_{j}(\Omega)\right|^{2}-\left|V_{j}(\Omega)\right|^{2}=1, \quad j=s, i \\
& U_{s}(\Omega) V_{i}(-\Omega)=U_{i}(-\Omega) V_{s}(\Omega)
\end{aligned}
$$

Most importantly, $U_{j}(\Omega)$ and $V_{j}(\Omega)$ diverge when approaching

$$
g=g_{\mathrm{thr}}=\frac{\pi}{2},
$$

the value of the parametric gain corresponding to the MOPO threshold in the CW pump regime [15].

\section{CHARACTERISTIC SPECTRAL BANDWIDTHS}

A Taylor expansion of the phase mismatch $\mathcal{D}(\Omega) \sqrt{6}$ in series of the frequency offset $\Omega$ gives:

$$
\begin{aligned}
\frac{\mathcal{D}(\Omega) l_{c}}{2} & =\frac{l_{c}}{2}\left(k_{s}^{\prime}+k_{i}^{\prime}\right) \Omega+\frac{l_{c}}{4}\left(k_{s}^{\prime \prime}-k_{i}^{\prime \prime}\right) \Omega^{2}+\cdots \\
& \simeq \frac{l_{c}}{2}\left(k_{s}^{\prime}+k_{i}^{\prime}\right) \Omega:=\tau_{\mathrm{gvs}} \Omega
\end{aligned}
$$

where $k_{j}^{\prime}, k_{j}^{\prime \prime}$ indicate derivatives of the wavenumbers $k_{j}(\Omega)$, calculated at the reference frequencies $\Omega=0$, and

$$
\tau_{\mathrm{gvs}}=\frac{1}{2}\left[\frac{l_{c}}{v_{g s}}+\frac{l_{c}}{v_{g i}}\right] \equiv \Omega_{\mathrm{gvs}}{ }^{-1}
$$

is a time scale characteristic of counterpropagating interactions, involving the sum of the inverse group velocities $v_{g j}=1 / k_{j}^{\prime}$. This long time scale roughly corresponds to the maximal delay that may occur between the exits of two twins down-converted from the same pump photon, and is on the order of the transit time of light along the slab because they appear at its opposite sides [7, 9]. Its inverse $\Omega_{\mathrm{gvs}}$ is responsible the narrow width of the spectrum of downconverted light, both below [6, 7, 9, 14, and above the MOPO threshold [5. The first term in the Taylor expansion (10) is by far the dominant one, so that the linear approximation for the phase mismatch in Eq. (11) is well justified.

Figure 3 shows an example of such spectra, calculated for various values of $g$ below the MOPO threshold. Precisely, it shows the spectral density $\left|V_{s}(\Omega)\right|^{2}=\left|V_{i}(-\Omega)\right|^{2}$, such that

$$
\left\langle\hat{A}_{s}^{\text {†out }}(\Omega) \hat{A}_{s}^{\text {out }}\left(\Omega^{\prime}\right)\right\rangle=\left\langle\hat{A}_{i}^{\text {tout }}(-\Omega) \hat{A}_{i}^{\text {out }}\left(-\Omega^{\prime}\right)\right\rangle=\delta\left(\Omega-\Omega^{\prime}\right)\left|V_{s}(\Omega)\right|^{2}
$$



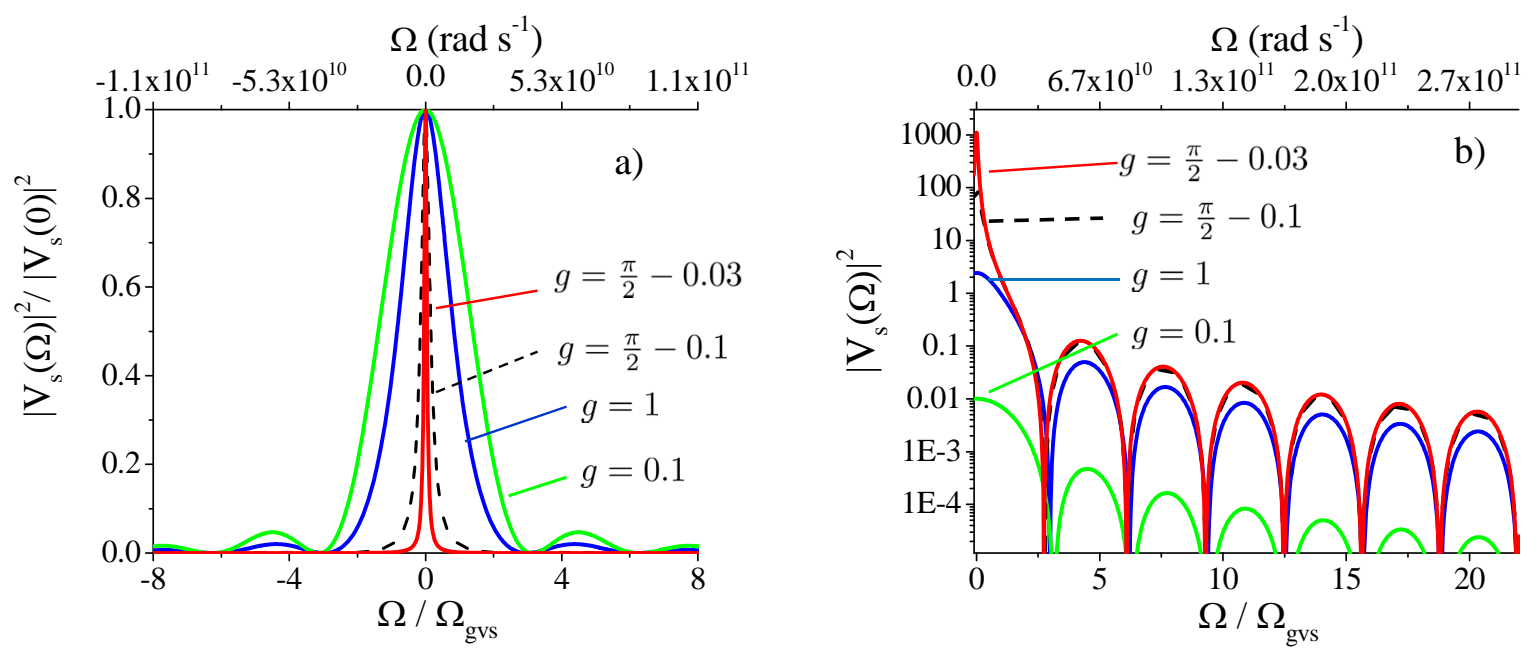

FIG. 3: (Color online) Intensity spectra $\left|V_{s}(\Omega)\right|^{2} \sqrt{13}$ ), plotted for different values of the gain $g$, as a function of $\Omega / \Omega_{\mathrm{gvs}}($ bottom axis) and of the frequency in a PPLN slab poled for the counterpropagating type 0 interaction. In a) the spectra are normalized to their peak value, in b) they are shown in logarithmic scale. $\lambda_{p}=771 \mathrm{~nm}, \lambda_{s}=\lambda_{i}=1542 \mathrm{~nm}, \Lambda=212.7 \mathrm{~nm}, l_{c}=1 \mathrm{~cm}$.

Notice that the singular Dirac $\delta$ in this equation is an artifact coming from assuming a monochromatic pump, of infinite duration, and would disappear with a proper regularization. The plots in Fig 3 have been calculated for a periodically poled Lithium Niobate (PPLN) slab of $1 \mathrm{~cm}$ length, pumped at $\lambda_{p}=771 \mathrm{~nm}$, with a poling period $\Lambda=212.7 \mathrm{~nm}$, suitable to phase match the degenerate type 0 interaction at $\lambda_{s}=\lambda_{i}=1542 \mathrm{~nm}$. The wave-numbers were evaluated using the complete Sellmeier relations in [16. Actually, these plots as a function of the normalized frequency are approximately valid for any material and tuning condition of the MOPO, because in the linear approximation (11) the spectra are just function of $\Omega / \Omega_{\text {gvs }}$ As described in detail in Ref. [9], they show that well below the MOPO threshold parametric generation occurs in the narrow bandwidth $\Omega_{\text {gvs }}$, and that on approaching threshold the bandwidth shrinks $[$ fig 3 a $)]$, while the mean photon number grows[fig $3 \mathrm{~b})]$, ideally to infinite $[9]$.

On the other side, an analogous Taylor expansion of the propagation phase $\beta(\Omega)$ in Eq. (7) gives

$$
\begin{aligned}
\beta(\Omega) & =\left(k_{s}^{\prime}-k_{i}^{\prime}\right) \frac{l_{c}}{2} \Omega+\frac{l_{c}}{4}\left(k_{s}^{\prime \prime}+k_{i}^{\prime \prime}\right) \Omega^{2}+\cdots \\
& =\tau_{\mathrm{gvm}} \Omega+\tau_{\mathrm{gvd}}{ }^{2} \Omega^{2}+\cdots
\end{aligned}
$$

where

$$
\begin{aligned}
& \tau_{\mathrm{gvm}}=\frac{1}{2}\left[\frac{l_{c}}{v_{g s}}-\frac{l_{c}}{v_{g i}}\right] \equiv \Omega_{\mathrm{gvm}}{ }^{-1} \\
& \tau_{\mathrm{gvd}}=\sqrt{\frac{l_{c}}{4}\left(k_{s}^{\prime \prime}+k_{i}^{\prime \prime}\right)} \equiv \Omega_{\mathrm{gvd}}{ }^{-1}
\end{aligned}
$$

are short time scales, linked respectively to the group velocity mismatch between the two waves and to the dispersion of the group velocities. Notice that these two time scales are also characteristic of the co-propagating interactions, and are short compared to $\tau_{\mathrm{gvs}}$. Taking the example of the PPLN of Fig. 3 we have: $\tau_{\mathrm{gvs}}=74.9 \mathrm{ps}\left(\Omega_{\mathrm{gvs}}=\right.$ $\left.1.336 \times 10^{10} \mathrm{rad} \mathrm{s}^{-1}\right) ; \tau_{\mathrm{gvm}}=0 ; \tau_{\mathrm{gvd}}=0.016 \mathrm{ps}\left(\Omega_{\mathrm{gvd}}=4.45 \times 10^{13} \mathrm{rad} \mathrm{s}^{-1}\right)$. As a consequence, the associated bandwidth are broad, as compared to the MOPO bandwidth $\Omega_{\mathrm{gvs}}$, and the phase $\beta(\Omega)$ has a very slow variation inside the whole bandwidth of emission, a peculiar circumstance of the counterpropagating interaction, that as we shall see has important consequences on the squeezing.

\section{GENERAL PROPERTIES OF THE OUTPUT STATE}

Several properties of the quantum state of the MOPO below threshold are common to any PDC process, and in general to all linear processes where particles are generated in pairs, because they depend solely on the form of the 
Bogoliubov input -output transformation (2). This section will review these properties, and outline their link to the coefficients of the transformation (2).

In order to avoid formal difficulties coming to the continuum of modes, in the following of this section we assume some form of discretization of frequencies, e.g by taking a finite quantization time interval $T$, so that $\Omega \rightarrow \Omega_{n}=\frac{2 \pi n}{T}$ becomes a discrete set of frequencies .

In the monochromatic pump approximation, parametric coupling exists only between frequency-conjugate spectral components of the signal and idler fields, say $\hat{A}_{s}(\Omega)$ and $\hat{A}_{i}(-\Omega)^{1}$, as expressed by the Bogolubov transformation (2) The unitarity of such transformation constrains its coefficients $U_{j}(\Omega) V_{j}(\Omega)$ to obey the conditions $(8)$, so that they can be recast in terms of fewer parameters. In particular, by introducing the squeezing parameter $r(\bar{\Omega})$ and the squeezing angle $\theta(\Omega)$

$$
\begin{aligned}
& \left|U_{s}(\Omega)\right|=\left|U_{i}(-\Omega)\right|:=\cosh [r(\Omega)] \\
& \left|V_{s}(\Omega)\right|=\left|V_{i}(-\Omega)\right|:=\sinh [r(\Omega)] \\
& \arg \left[U_{s}(\Omega) V_{i}(-\Omega)\right]=\arg \left[U_{i}(-\Omega) V_{s}(\Omega)\right]:=2 \theta(\Omega),
\end{aligned}
$$

the input-output transformation can be rewritten in the standard form of a two-mode squeeze transformation:

$$
\begin{aligned}
& \hat{A}_{s}^{\text {out }}(\Omega)=\cosh [r(\Omega)] \hat{B}_{s}^{\text {in }}(\Omega)+e^{2 i \theta(\Omega)} \sinh [r(\Omega)] \hat{B}_{i}^{\text {in } \dagger}(-\Omega) \\
& \hat{A}_{i}^{\text {out }}(-\Omega)=\cosh [r(\Omega)] \hat{B}_{i}^{\text {in }}(-\Omega)+e^{2 i \theta(\Omega)} \sinh [r(\Omega)] \hat{B}_{s}^{\text {in } \dagger}(\Omega)
\end{aligned}
$$

where the new input operators are are just phase rotated versions of the original input operators: $\hat{B}_{s}^{\mathrm{in}}(\Omega)=$ $e^{i \varphi_{u s}(\Omega)} \hat{A}_{s}^{\mathrm{i} n}(\Omega), \hat{B}_{i}^{\mathrm{i} n}(-\Omega)=e^{i \varphi_{u i}(-\Omega)} \hat{A}_{i}^{\mathrm{i} n}(-\Omega)$, with $\varphi_{u j}(\Omega)=\arg \left[U_{j}(\Omega)\right]$. Clearly, such a rotation has no effect on the input vacuum state. The generator of the transformation [21] is the two-mode squeeze operator (see e.g Refs. [17, [18])

$$
\hat{R}(\xi)=e^{\sum_{\Omega}\left[\xi(\Omega) \hat{A}_{s}^{\dagger}(\Omega) A_{i}^{\dagger}(-\Omega)-\xi^{*}(\Omega) \hat{A}_{s}(\Omega) A_{i}(-\Omega)\right]}, \quad \xi(\Omega)=r(\Omega) e^{2 i \theta(\Omega)},
$$

such that Eq. 21 can be recast as:

$$
\begin{aligned}
& \hat{A}_{s}^{\text {out }}(\Omega)=\hat{R}^{\dagger}(\xi) \hat{B}_{s}^{\text {in }}(\Omega) \hat{R}(\xi) \\
& \hat{A}_{i}^{\text {out }}(-\Omega)=\hat{R}^{\dagger}(\xi) \hat{B}_{i}^{\text {in }}(-\Omega) \hat{R}(\xi)
\end{aligned}
$$

If the same transformation, instead of acting on the input operators, is applied to the input vacuum state, it generates at the output of the crystal the entangled state of twin beams. This can be written as the tensor product of states belonging to subspaces at fixed $\Omega$ :

$$
|\Psi\rangle^{\mathrm{out}}=\prod_{\Omega}|\psi\rangle_{\Omega}^{\mathrm{o} u t}
$$

where $|\psi\rangle_{\Omega}^{\text {out }}$ indicates the state of the two coupled signal and idler modes at frequencies $\omega_{s}+\Omega$ and $\omega_{i}-\Omega$. It can be calculated as

$$
\begin{aligned}
|\psi\rangle_{\Omega}^{\mathrm{out}} & =\hat{R}(\xi)|0\rangle_{\Omega, s}|0\rangle_{-\Omega, i}=\sum_{N=0}^{+\infty} c_{N}(\Omega)|N\rangle_{\Omega, s}|N\rangle_{-\Omega, i}, \\
c_{N}(\Omega) & =\frac{\tanh [r(\Omega)]^{N}}{\cosh [r(\Omega)]} e^{2 i N \theta(\Omega)},
\end{aligned}
$$

where $|N\rangle_{\Omega, s}$, and $|N\rangle_{-\Omega, i}$ denote Fock states, with $\mathrm{N}$ photons in each of the two modes. This is the well known two-mode squeeze state, which is an eigenstate with null eigenvalue of the difference of the signal/idler photon numbers at $\pm \Omega$ :

$$
\left[\hat{A}_{s}^{\dagger}(\Omega) \hat{A}_{s}(\Omega)-\hat{A}_{i}^{\dagger}(-\Omega) \hat{A}_{i}(-\Omega)\right]|\psi\rangle_{\Omega}^{\text {out }}=0
$$

\footnotetext{
${ }^{1}$ Notice that for a finite pump bandwidth, coupling will exists in a range of frequencies proportional to the pump bandwidth
} 
This implies the existence of a perfect correlation between the photon numbers detected at each pair of conjugate frequencies of the twin beams. In Sec. VI we shall come back to a more operative definition of the photon number correlation in the spectro-temporal continuum.

Besides the photon-number correlation, this state is also well known to to display a noteworthly EPR-type of correlation between two non-commuting quadrature operators of the signal and idler field [19, 20. Precisely, if one focuses on a pair of frequency-conjugate spectral components $\omega_{s}+\Omega, \omega_{i}-\Omega$, and introduces their sum and difference

$$
\hat{c}_{ \pm}(\Omega)=\frac{\hat{A}_{s}^{\text {out }}(\Omega) \pm A_{i}^{\text {out }}(-\Omega)}{\sqrt{2}},
$$

then the transformation (2) decouples into two independent squeeze transformations

$$
\begin{aligned}
& \hat{c}_{+}(\Omega)=\cosh [r(\Omega)] \hat{B}_{+}^{\mathrm{in}}(\Omega)+e^{2 i \theta(\Omega)} \sinh [r(\Omega)] \hat{B}_{+}^{\mathrm{in} \dagger}(\Omega) \\
& \hat{c}_{-}(\Omega)=\cosh [r(\Omega)] \hat{B}_{-}^{\mathrm{in}}(\Omega)-e^{2 i \theta(\Omega)} \sinh [r(\Omega)] \hat{B}_{-}^{\mathrm{in} \dagger}(\Omega) .
\end{aligned}
$$

where $\hat{B}_{ \pm}^{\text {in }}$ are independent modes in the vacuum state, defined in obvious way as the sum and difference of the input signal-idler modes. Thus the \pm modes, which combine frequency conjugate signal and idler spectral components (notice that this slightly differs to what is in practice done in a measurement, see next section) are independent and individually squeezed. The two parameters $\theta$ and $r$, defined by Eq. (19), determine the phase angle at which noise reduction occurs, and the maximum level of squeezing achievable, respectively. Namely, for the difference mode $\hat{c}_{-}(\Omega)$, best squeezing occurs for the field quadrature at angle $\theta(\Omega)$, while for the sum mode $\hat{c}_{+}(\Omega)$ it occurs in the orthogonal quadrature at angle $\theta(\Omega)+\pi / 2$. At these angles, quantum noise is reduced below the shot noise value " 1 " by an amount

$$
e^{-2 r(\Omega)}=\left|U_{s}(\Omega)\right|-\left|V_{i}(-\Omega)\right|^{2}=\frac{1}{\left|U_{s}(\Omega)\right|+\left|V_{i}(-\Omega)\right|^{2}}
$$

As well know, this means that the signal and idler quadratures at angle $\theta(\Omega)$ are correlated, while at the same time the orthogonal quadratures at angle $\theta(\Omega)+\pi / 2$ are anticorrelated. The degree of simultaneous correlation/anticorrelation in the orthogonal quadratures can be large enough to provide a realization of the original EPR paradox [19, 20].

\section{QUADRATURE CORRELATION IN THE MOPO BELOW THRESHOLD}

In order to characterize the squeezing and EPR correlation generated in specific case of the MOPO, let us come back to the continuum of frequencies and introduce a definition of the phase sensitive noise suitable for measurements. To this end, we consider the signal $(j=s)$ and idler $(j=i)$ quadrature operators in the time domain:

$$
\begin{aligned}
\hat{X}_{j}(t) & =\hat{A}_{j}^{\mathrm{out}}(t) e^{-i \phi_{j}}+\hat{A}_{j}^{\mathrm{out} \dagger}(t) e^{i \phi_{j}}, \\
\hat{Y}_{j}(t) & =-i\left[\hat{A}_{j}^{\mathrm{out}}(t) e^{-i \phi_{j}}-\hat{A}_{j}^{\mathrm{out} \dagger}(t) e^{i \phi_{j}}\right]
\end{aligned}
$$

which, by varying the phase-angles $\phi_{j}$, span all the classical phase-space of the harmonic oscillator, remaining orthogonal $\hat{X} \rightarrow \hat{Y}$ for $\phi_{j} \rightarrow \phi_{j}+\frac{\pi}{2}$. Next, we introduce proper combinations of these signal and idler operators:

$$
\hat{X}_{-}(t)=\frac{\hat{X}_{s}(t)-\hat{X}_{i}(t)}{\sqrt{2}} \quad, \hat{Y}_{+}(t)=\frac{\hat{Y}_{s}(t)+\hat{Y}_{i}(t)}{\sqrt{2}}
$$

which basically represent the quadrature operators of the modes $\hat{c}_{-}, \hat{c}_{+}$in Eq. (28). The quadrature noise in the sum and difference modes is then characterized by the spectra of squeezing

$$
\begin{aligned}
& \Sigma_{-}(\Omega)=\int_{-\infty}^{+\infty} d \tau e^{i \Omega \tau}\left\langle\delta \hat{X}_{-}(t) \hat{\delta} X_{-}(t+\tau)\right\rangle \\
& \Sigma_{+}(\Omega)=\int_{-\infty}^{+\infty} d \tau e^{i \Omega \tau}\left\langle\delta \hat{Y}_{+}(t) \hat{\delta} Y_{+}(t+\tau)\right\rangle
\end{aligned}
$$

These quantities describe the degree of correlation ("-" sign) or anticorrelation ("+" sign) existing between the field quadrature operators of the twin beams at the two crystal output faces. With our definitions, the value "1" represents 
the shot noise level, which corresponds to two uncorrelated coherent light beams. In the case of signal and idler fields with the same central frequency, one may also think of physically recombining the two counterpropagating light beams, in order to produce two independently squeezed beams.

After some calculations, based on the input-output relations (2), we obtain

$$
\begin{aligned}
\Sigma_{ \pm}(\Omega) & =\frac{1}{2}[\mathcal{F}(\Omega)+\mathcal{F}(-\Omega)] \\
\mathcal{F}(\Omega) & =\left|U_{s}(\Omega)-V_{i}^{*}(-\Omega) e^{i\left(\phi_{s}+\phi_{i}\right)}\right|^{2}
\end{aligned}
$$

The two symmetric spectral components $\mathcal{F}(\Omega), \mathcal{F}(-\Omega)$ can be shown to represent the noise in the two sidebands modes $\hat{c}_{ \pm}(\Omega)$, and $\hat{c}_{ \pm}(-\Omega)$ [10]. As described in [10], the best squeezing is achieved by choosing the phase-angles as

$$
\begin{aligned}
\phi_{s}+\phi_{i}=2 \theta( \pm \Omega) & =k_{s} l_{c}+\phi_{p}+\arg [\operatorname{sinc} \gamma( \pm \Omega)]+\beta( \pm \Omega) \\
& \simeq k_{s} l_{c}+\phi_{p}+\arg [\operatorname{sinc} \gamma(\Omega)]
\end{aligned}
$$

where the last line uses the fact that the phase $\beta$ is almost constant over the entire MOPO spectrum, and the linear approximations (11), which implies $\gamma(\Omega)=\sqrt{g^{2}+\frac{\Omega^{2}}{\Omega_{g v s}^{2}}}=\gamma(-\Omega){ }^{2}$ With this choice
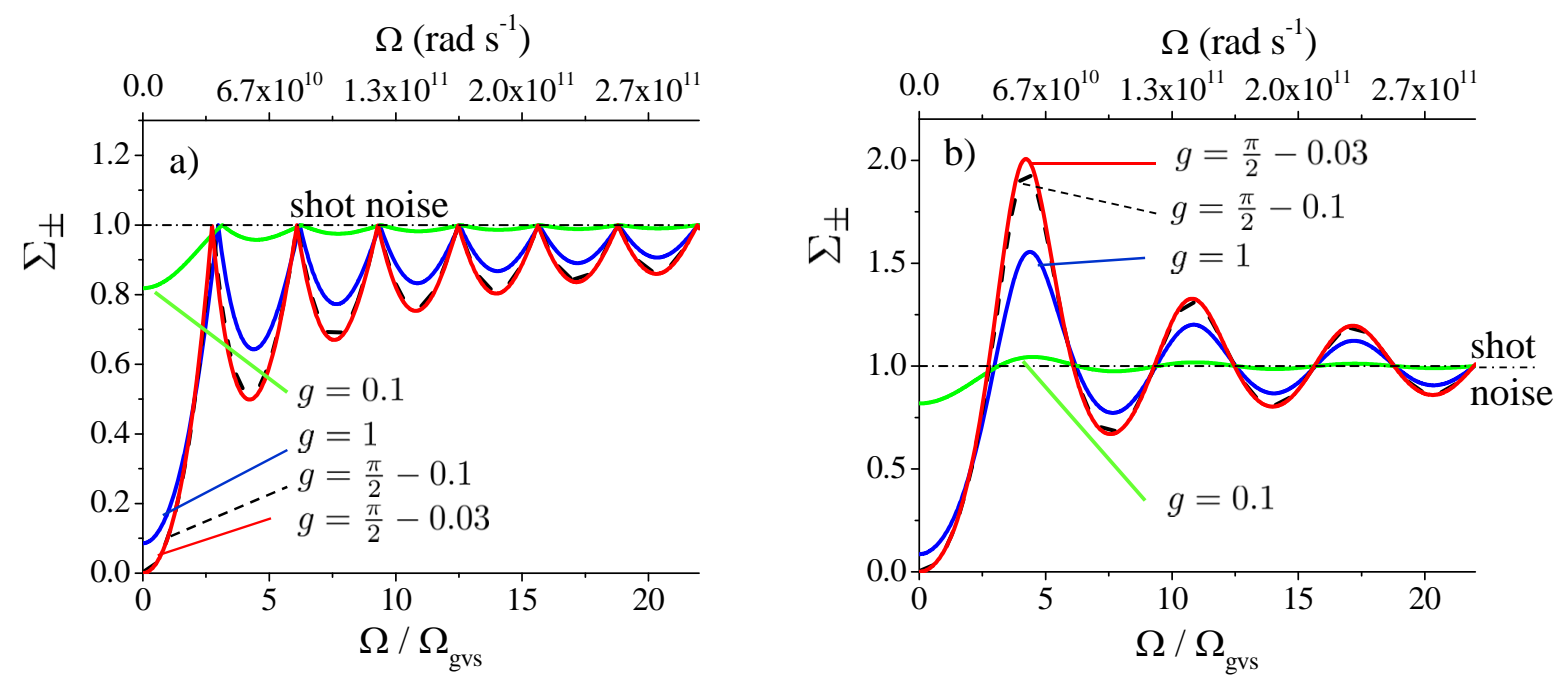

FIG. 4: (Color online) Squeezing spectra $\Sigma_{ \pm}(\Omega)(33)$ in the sum or difference modes, and degree of EPR correlation between field quadratures of the MOPO twin beams,plotted for different values of the gain $g$, as a function of the normalized frequency $\Omega / \Omega_{\text {gvs }}$ (bottom axis) and of the frequency (upper axis) in a PPLN counterpropagating slab. In a) the quadrature angles are optimized for best squeezing [Eq. [36] ]. In b) they are fixed as $\phi_{s}+\phi_{i}=k_{s} l_{c}+\phi_{p}$. Parameters as in Fig 3

$$
\Sigma_{ \pm}(\Omega) \rightarrow\left[\left|U_{s}(\Omega)\right|-\left|V_{i}(-\Omega)\right|\right]^{2}=e^{-2 r(\Omega)}
$$

reaches its minimum value at any frequency, and the noise never goes above the shot noise level "1", as shown by Fig 4 a. Fig 4 b shows instead the degree of squeezing/EPR correlation when the phase-angles are fixed as

$$
\phi_{s}+\phi_{i}=2 \theta(0)=k_{s} l_{c}+\phi_{p}
$$

As described in detail Ref. [10, we see that when the MOPO threshold is approached the level of squeezing and EPR correlation become asymptotically perfect, showing a behaviour completely analogous to that of a cavity OPO below threshold, which is the standard source of squeezed light. Good levels of EPR correlation are present even quite far from threshold, having for example a $90 \%$ squeezing for $g=1$, which is $36 \%$ below the threshold.

\footnotetext{
${ }^{2}$ Alternatively, as explained in Ref.[10] in the presence of GVM, a further optimization can be done by introducing a delay $\Delta t=\tau_{\mathrm{gvm}}$ between the detection of the twin beams, which compensate the offset between their exit times.
} 
Most important, as evident from a comparison between Fig $4 \mathrm{~b}$ and the MOPO spectra in Fig. 3, excellent levels of squeezing and EPR correlation can be obtained inside the entire emission bandwidth of the MOPO even when the phase angles are fixed as in Eq. (38) $\phi_{s}+\phi_{i}=2 \theta(0)$. This is important because in practice the detection will be performed mostly at fixed phase angles. In this case, the noise passes from below to above the shot noise at $|\Omega|=\Omega_{\mathrm{gvs}} \sqrt{\pi^{2}-g^{2}}$, i.e. at the point where the $\operatorname{sinc} \gamma(\Omega)$ changes sign. Thus the bandwidth of squeezing $\Delta \Omega_{\mathrm{squeeze}}=\Omega_{\mathrm{gvs}} \sqrt{\pi^{2}-g^{2}}$ remains approximately constant in the neighborhood of the threshold $\Delta \Omega_{\text {squeeze }} \approx 2.7 \Omega_{\mathrm{gvs}}$.

It is interesting to compare this behavior with what can be obtained in the standard single-pass co-propagating configuration. Within the monochromatic and undepleted pump approximations this setup can be modeled by the same input-output Bogoliubov tranformation (2), with the obvious difference that the output field operators appear in this case on the same face of the slab. The coefficients $U_{j}$ and $V_{j}$ have to be substituted by those calculated for the co-propagating parametric equations, and can be for example found in Refs. [18, 21] (the transverse wave-vector appearing there has to be set to $\vec{q}=0$ in order to describe collinear propagation). The squeezing spectra and the intensity spectra are then calculated from the same Eqs. (33) and (13), with the proper coefficients inserted. We can take the example of a $1 \mathrm{~cm}$ PPLNslab, pumped at $771 \mathrm{~nm}$, similarly to the MOPO case, but with a long poling poling period $\Lambda=18.8 \mu \mathrm{m}$, chosen to phase match the co-propagating degenerate type 0 interaction. Results for the squeezing spectra and for the intensity spectra are shown in figures 5 and 6 , respectively. The relevant spectral scale is in this case the ultrabroad dispersion bandwidth $\Omega_{\mathrm{gvd}}$, because the phase mismatch for the co-propagating configuration is given by

$$
\begin{aligned}
\Delta(\Omega) \frac{l_{c}}{2} & =\left[k_{s}(\Omega)+k_{i}(-\Omega)-k_{p}+k_{G}\right] \frac{l_{c}}{2}=\left(k_{s}^{\prime}-k_{i}^{\prime}\right) \frac{l_{c}}{2} \Omega+\frac{k_{s}^{\prime \prime}+k_{i}^{\prime \prime}}{2} \frac{l_{c}}{2} \Omega^{2}+\ldots \\
& =\tau_{\mathrm{gvm}} \Omega+\tau_{\mathrm{gvd}}{ }^{2} \Omega^{2} \rightarrow\left(\frac{\Omega}{\Omega_{\mathrm{gvd}}}\right)^{2}
\end{aligned}
$$

where the last result holds for the type 0 or type I interactions at degeneracy, where $\tau_{\mathrm{gvm}}=0$. The parameters $\tau_{\mathrm{gvm}}, \tau_{\mathrm{gvd}}$ and $\Omega_{\mathrm{gvd}}$ are the same as defined by Eqs. (16) and (17).

As well known, the fluorescence spectra are in this case ultrabroadband [see Fig. 66, because ruled by the dispersion bandwidth $\Omega_{\mathrm{gvd}}$, and tend to become slightly broader when the parametric gain increases (notice that $g$ in these figure is defined by Eq. (1), exactly in the same way as for the MOPO, but obviously there is no threshold value). In contrast, the squeezing spectra in Fig 5 exhibit noise reduction below the shot noise in a smaller bandwidth, which in particular shrinks dramatically as soon as the parametric gain grows above the value $g \simeq 1$ at which stimulated emission starts to be important. Then, at high gain, it would be difficult to observe squeezing in the whole fluorescence bandwidth.
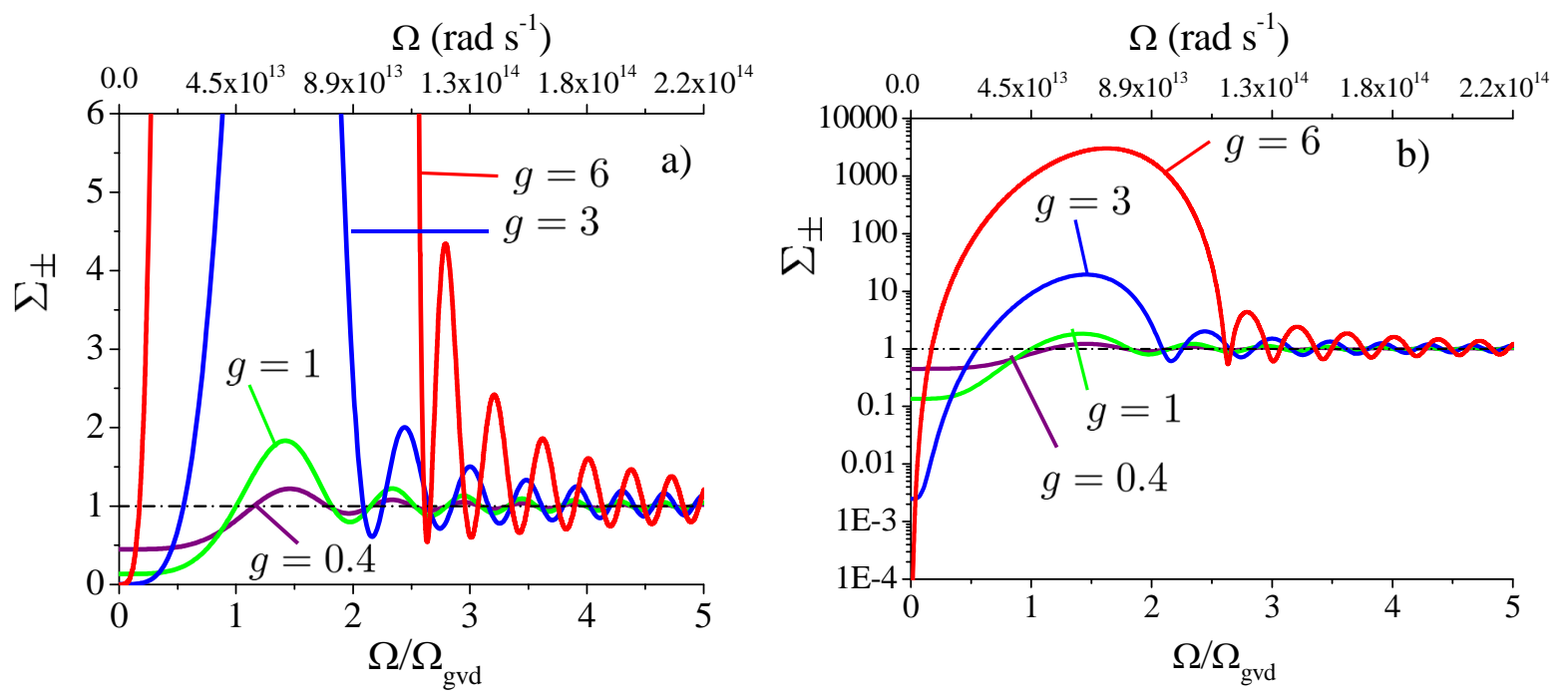

FIG. 5: (Color online) Co-propagating configuration: a) Squeezing spectra $\Sigma_{ \pm}(\Omega)(33)$, plotted for different values of the gain $g$, as a function of the normalized frequency $\Omega / \Omega_{\mathrm{gvd}}$ (bottom axis) and of the frequency (upper axis) in a PPLN co-propagating slab. The quadrature angles are fixed as $\left.\phi_{s}+\phi_{i}=\theta(0)=\left(k_{p}-k_{G}\right) l_{c}+\phi_{p} . \mathrm{b}\right)$ Same plot in logarithmic scale. $\lambda_{p}=771 \mathrm{~nm}$, $\lambda_{s}=\lambda_{i}=1542 \mathrm{~nm}, \Lambda=18.8 \mu \mathrm{m}, l_{c}=1 \mathrm{~cm}$.

This happens because the orientation of the squeezing ellipses varies rapidly with the frequency inside the broad PDC 

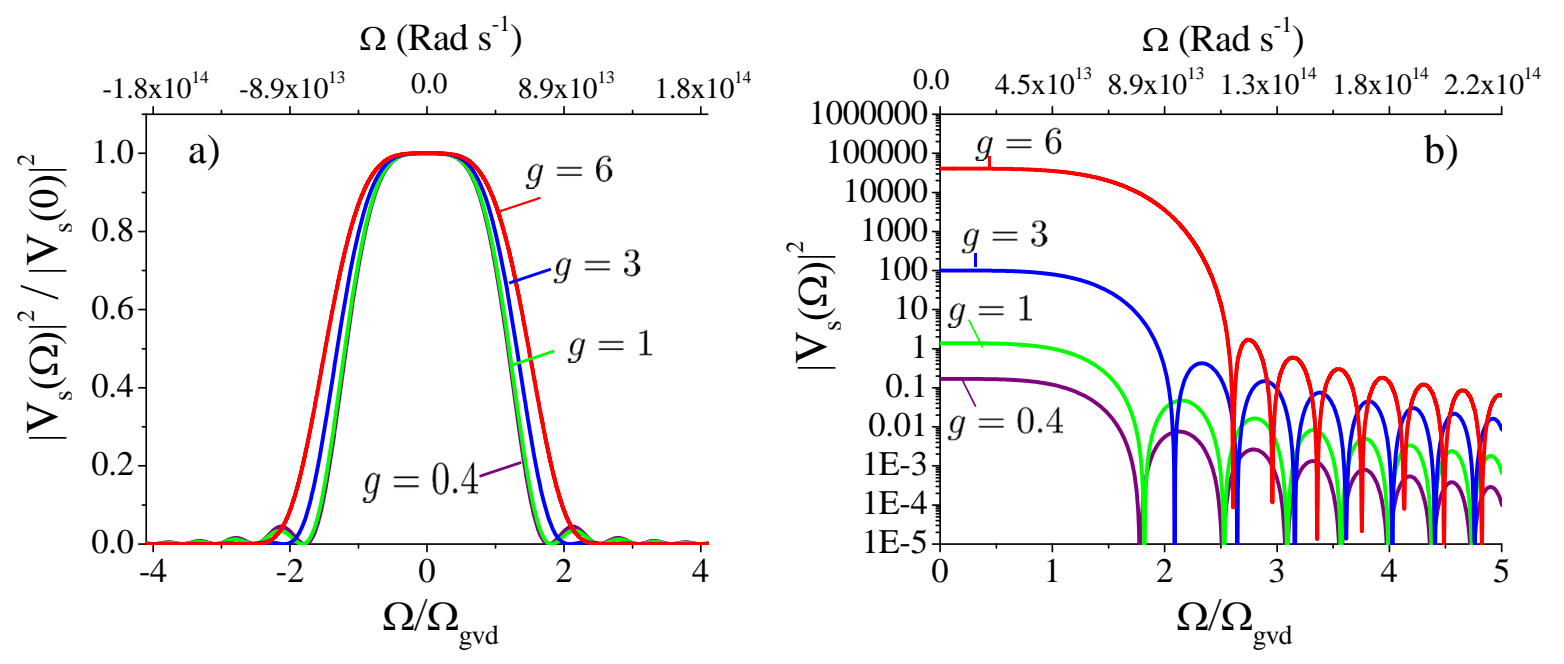

FIG. 6: (Color online)Co-propagating configuration: Intensity spectra $\left|V_{s}(\Omega)\right|^{2} \sqrt{13}$ ), plotted for different values of the gain $g$, as a function of $\Omega / \Omega_{\mathrm{gvd}}$ (bottom axis) and of $\Omega$ (upper axis) in a PPLN slab poled for the co-propagating type 0 interaction. Parameters as in figure 5

bandwidth. Calculations based on the explicit solution of the co-propagating parametric equations in [18, 21] show that in this case the angle at which best squeezing occurs is $\theta(\Omega)-\theta(0) \simeq\left(\frac{\Omega}{\Omega_{\text {gvd }}}\right)^{2} \frac{\tanh g}{g}$, thus varying on a similar scale $\simeq \Omega_{\mathrm{gvd}}$ as the spectrum. Notice that, when the phase-angles are fixed as $\phi_{s}+\phi_{i}=2 \theta(0)$, the detected squeezing spectra can be written as:

$$
\begin{aligned}
\Sigma_{ \pm}(\Omega) & =\left|U_{s}(\Omega)-V_{i}^{*}(-\Omega) e^{2 i \theta(0)}\right|^{2}=e^{-2 r(\Omega)}+2 \sinh [2 r(\Omega)] \sin ^{2}[\theta(\Omega)-\theta(0)] \\
& \approx e^{-2 r(\Omega)}+e^{2 r(\Omega)} \sin ^{2}[\theta(\Omega)-\theta(0)] \quad \text { for } r(\Omega) \gg 1
\end{aligned}
$$

For significant squeezing $r \geq 1$, as soon as the squeezing phase has a small change with respect to $\theta(0)$, a large excess noise (the second term at r.h.s) appears and degrades the squeezing.

This behaviour is in sharp contrast with the MOPO, where the orientation of the squeezing ellipses, defined by Eqs. (35) 36 varies slowly with the frequency, and remains constant inside the narrow MOPO bandwidth $\simeq \Omega_{\mathrm{gvs}}$. This can be seen as a consequence of the peculiar time scale $\tau_{\text {gvs }}$ involved in the counterpropagating interaction, which is much longer than the other time scales $\tau_{\mathrm{gvm}}, \tau_{\mathrm{gvd}}$ that characterize the co-propagation of light waves.

Notice that in the co-propagating case the bandwidth of squeezing, despite it shrinks with increasing gain, remains in any case ultrabroad, in the Thz region (see the upper scales in Figs. 4 and 5), and probably could be further enlarged by properly shaping the phase of the local oscillator.

\section{INTENSITY CORRELATION}

We now turn our attention to the quantum correlation between the intensities of the MOPO twin beams.

As a trivial consequence of the pairwise generation of photons, when the MOPO intensities are collected for a long enough time, the detected photon numbers will be identical not only in the mean values, but also in their quantum fluctuations. A good question is then how long one has to detect before the photon-number difference shows fluctuations below the shot-noise that represents the classical limit? Our curiosity in this sense is driven by the findings of Ref. [9]. Here it was shown that in the spontaneous regime the MOPO twin photons are correlated over the time $\tau_{\text {corr }}=\tau_{\mathrm{gvs}}$, which simply reflects the fact that twin photons originating from the same pump photon exit the crystal at most delayed by their transit time across the slab. In the stimulated regime, however, the correlation becomes long ranged, and ideally $\tau_{c o r r} \rightarrow \infty$ as threshold is approached, as a consequence of a combination of stimulated emission and back-propagation. Then, we ask ourselves whether close to threshold an infinite detection time would be needed to observe sub-shot noise intensity fluctuations.

To this end, we consider the instantaneous intensity operators at the crystal output faces $\hat{I}_{j}(t)=\hat{A}_{j}^{\text {tout }}(t) \hat{A}_{j}^{\text {out }}(t)$ 
$(j=s, i)$. In order to describe their level of correlation, on the one side we introduce the intensity difference

$$
\hat{I}_{-}(t)=\hat{I}_{s}(t)-\hat{I}_{i}(t)
$$

and calculate its spectrum of fluctuations:

$$
\mathcal{V}_{-}(\Omega)=\int d \tau e^{i \Omega \tau}\left\langle\delta I_{-}(t) \delta I_{-}(t+\tau)\right\rangle
$$

On the other side, we also consider the photon-number operators that result from integrating the intensities over a finite detection time $T_{d}$ :

$$
\hat{N}_{j}=\int_{-\frac{T_{d}}{2}}^{\frac{T_{d}}{2}} d t \hat{I}_{j}(t)=\int_{-\frac{T_{d}}{2}}^{\frac{T_{d}}{2}} d t \hat{A}_{j}^{\dagger \text { out }}(t) \hat{A}_{j}^{\text {out }}(t) \quad j=s, i
$$

and evaluate the noise in their difference $\hat{N}_{-}=\hat{N}_{s}-\hat{N}_{i}$.

$$
\left\langle\left(\delta \hat{N}_{-}\right)^{2}\right\rangle=\left\langle\left(\delta \hat{N}_{s}-\delta \hat{N}_{i}\right)^{2}\right\rangle
$$

When these two quantities go below their shot-noise level, characterizing coherent light or classically correlated beams (e.g. generated by splitting thermal light on a beam splitter [22]), we can then talk of a quantum correlation of microscopic nature.

The two approaches are linked, because the variance 44 can be written in terms of the spectrum 42 as

$$
\begin{aligned}
\left\langle\left(\delta \hat{N}_{-}\right)^{2}\right\rangle & =\int_{\frac{-T_{d}}{2}}^{\frac{T_{d}}{2}} d t \int_{\frac{-T_{d}}{2}}^{\frac{T_{d}}{2}} d t^{\prime}\left\langle\delta \hat{I}_{-}(t) \delta \hat{I}_{-}\left(t^{\prime}\right)\right\rangle \\
& =T_{d}^{2} \int \frac{d \Omega}{2 \pi} \operatorname{sinc}^{2}\left(\frac{T_{d} \Omega}{2}\right) \mathcal{V}_{-}(\Omega)
\end{aligned}
$$

For an infinite detection time $T_{d} \rightarrow \infty,\left\langle\left(\delta N_{-}\right)^{2}\right\rangle \rightarrow T_{d} \mathcal{V}_{-}(\Omega=0)=0$. The same holds true when the detection time largely exceeds the inverse bandwidth of $\mathcal{V}_{-}(\Omega)$, because in that case the sinc function behaves as a $\delta$-function under the integral. Thus we expect that the variance 444 approaches zero for detection time $T_{d}$ longer than the inverse of the bandwidth of the spectrum 42 .

We start by calculating the spectrum of $\hat{I}_{-}$. To this end, we express the temporal correlation function of $I_{-}(t)$ in terms of the self- and cross-correlation of the intensities of the two fields

$$
\left\langle\delta \hat{I}_{-}(t) \delta \hat{I}_{-}(t+\tau)\right\rangle=G_{s s}^{(2)}(t, t+\tau)+G_{i i}^{(2)}(t, t+\tau)-G_{s i}^{(2)}(t, t+\tau)-G_{s i}^{(2)}(t, t-\tau),
$$

where

$$
G_{j l}^{(2)}\left(t, t^{\prime}\right):=\left\langle\delta \hat{I}_{j}(t) \delta \hat{I}_{l}\left(t^{\prime}\right)\right\rangle \quad j, l=i, s .
$$

In Eq. 47. we used the indentity $G_{i s}^{(2)}\left(t, t^{\prime}\right)=G_{s i}^{(2)}\left(t^{\prime}, t\right)$, and the fact that $G_{j l}^{(2)}\left(t, t^{\prime}\right)$ depends only on the time difference $t-t^{\prime}$ under stationary conditions. Since the model is linear, the fourth order field moments contained in the correlation functions $G_{j l}^{(2)}$ can be factorized into second-order field moments according to (see e.g. 23])

$$
\begin{aligned}
& G_{j j}^{(2)}\left(t, t^{\prime}\right)=\left\langle\delta \hat{I}_{j}(t) \delta \hat{I}_{j}\left(t^{\prime}\right)\right\rangle=\delta\left(t-t^{\prime}\right)\left\langle I_{j}\right\rangle+\left|\left\langle\hat{A}_{j}^{\dagger \text { out }}(t) \hat{A}_{j}^{\text {out }}\left(t^{\prime}\right)\right\rangle\right|^{2} \\
& G_{s i}^{(2)}\left(t, t^{\prime}\right)=\left\langle\delta \hat{I}_{s}(t) \delta \hat{I}_{i}\left(t^{\prime}\right)\right\rangle=\left|\left\langle\hat{A}_{s}^{\text {out }}(t) \hat{A}_{i}^{\text {out }}\left(t^{\prime}\right)\right\rangle\right|^{2}
\end{aligned}
$$

The second order field moments can be easily calculated from the input-output relations 22):

$$
\begin{aligned}
\left\langle\hat{A}_{s}^{\dagger \text { out }}(t) \hat{A}_{s}^{\text {out }}\left(t^{\prime}\right)\right\rangle & =\int \frac{d \Omega}{2 \pi} e^{i \Omega\left(t-t^{\prime}\right)}\left|V_{s}(\Omega)\right|^{2}=\left\langle\hat{A}_{i}^{\dagger \text { out }}\left(t^{\prime}\right) \hat{A}_{i}^{\text {out }}(t)\right\rangle \\
\left\langle\hat{A}_{s}^{\text {out }}(t) \hat{A}_{i}^{\text {out }}\left(t^{\prime}\right)\right\rangle & =\int \frac{d \Omega}{2 \pi} e^{-i \Omega\left(t-t^{\prime}\right)} U_{s}(\Omega) V_{i}(-\Omega)
\end{aligned}
$$


The first term at r.h.s. of Eq. (49) represents the shot-noise contribution, where the signal and idler mean intensities are

$$
\left\langle\hat{I}_{s}\right\rangle=\int \frac{d \Omega}{2 \pi}\left|V_{s}(\Omega)\right|^{2}=\int \frac{d \Omega}{2 \pi}\left|V_{i}(\Omega)\right|^{2}=\left\langle\hat{I}_{i}\right\rangle
$$

After some manipulations the noise spectrum can then be written as

$$
\mathcal{V}_{-}(\Omega)=\int \frac{d \Omega^{\prime}}{2 \pi}\left|U_{s}\left(\Omega^{\prime}\right) V_{i}^{*}\left(-\Omega-\Omega^{\prime}\right)-U_{s}\left(\Omega+\Omega^{\prime}\right) V_{i}^{*}\left(-\Omega^{\prime}\right)\right|^{2}
$$

This result is general to all processes of photon-pair generation, because it uses only the Bogoliubov transformation (2), and shows that $\mathcal{V}_{-}$vanishes identically at zero frequency $\Omega=0$. Following the discussion after Eq. 46), as anticipated, this implies that the noise in the photon number difference (44) also vanishes for an infinite detection time.

More insight into the MOPO case can be gained by inserting in Eq. (54) the explicit expression of the $U_{j}, V_{j}$ [Eq. [3] ], with which the spectrum of $I_{-}$becomes

$$
\begin{aligned}
\mathcal{V}_{-}(\Omega)=g^{2} \int \frac{d \Omega^{\prime}}{2 \pi}\left\{\left|\phi\left(\Omega^{\prime}\right)\right|^{2}\left|\phi\left(\Omega+\Omega^{\prime}\right)\right|^{2} \mid\right. & \operatorname{sinc}\left[\gamma\left(\Omega+\Omega^{\prime}\right)\right]+ \\
& \left.-\left.\operatorname{sinc}\left[\gamma\left(\Omega^{\prime}\right)\right] e^{i \beta\left(\Omega+\Omega^{\prime}\right)} e^{-i \beta\left(\Omega^{\prime}\right)}\right|^{2}\right\}
\end{aligned}
$$

Figure 7 plots the spectrum $\mathcal{V}_{-}(\Omega)$ of the intensity difference, normalized to the shot noise level $S N_{-} \equiv\left\langle I_{s}\right\rangle+\left\langle I_{i}\right\rangle$, evaluated through the numerical integration of the exact relation (55) for different values of the parametric gain $g$. We see that the signal and idler intensities display a sub-shot noise correlation within a bandwidth $\sim 2.5 \Omega_{\text {gvs }}$ both in the spontaneous and in the stimulated regime.

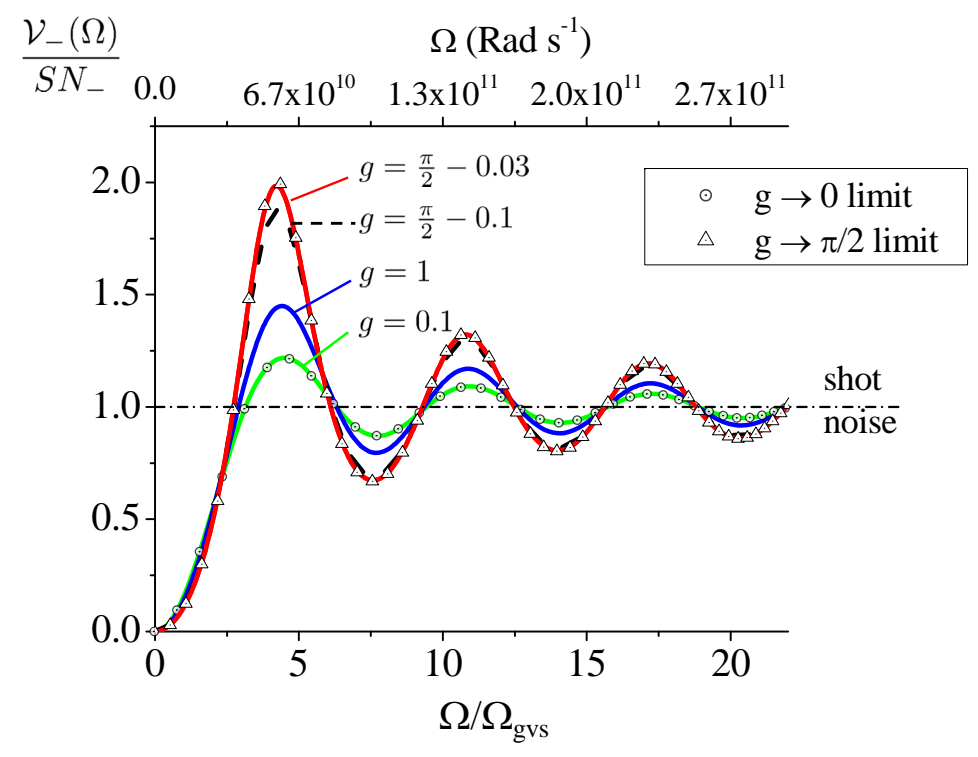

FIG. 7: Spectrum of $I_{-}$normalized to the shot-noise, in a PPLN counterpropagating slab, as evaluated from numerical integration of Eq. 55. The disc and the triangle symbols plot the approximated expressions in the spontaneous regime 62 and close to threshold 60, respectively. Parameters as in Fig 3

Eq. 55 can be used to obtain analytical estimations of the spectrum in the two limiting cases $g \rightarrow \frac{\pi}{2}$ (close to threshold) and $g \rightarrow 0$ (spontaneous regime) .

Close to threshold, for $g \rightarrow \frac{\pi}{2}$ and for values of $\Omega$ not too far from $\Omega=0$, the function $|\phi|^{2}$ appearing under the integral in Eq. (55) can be approximated as

$$
|\phi(\Omega)|^{2} \simeq \frac{g^{2}+\tilde{\Omega}^{2}}{g^{2} \sin ^{2} \epsilon+\tilde{\Omega}^{2}} \quad \text { for } \epsilon:=\frac{\pi}{2}-g \rightarrow 0
$$


where $\tilde{\Omega}=\frac{\Omega}{\Omega_{\mathrm{gvs}}}$. This is a rational function with two simple complex poles at $\tilde{\Omega}= \pm i g \sin \epsilon$, a result that can be used to calculate the integral in Eq- $\sqrt[54]{54}$ with a contour integration in the complex plane. As a result

$$
\mathcal{V}_{-}(\Omega) \simeq \frac{g \Omega_{\mathrm{gvs}}}{2 \sin \epsilon}\left[|\phi(\Omega)|^{2}\left|e^{i \beta(\Omega)}-g \operatorname{sinc}[\gamma(\Omega)]\right|^{2}+(\Omega \rightarrow-\Omega)\right] \quad \text { for } \epsilon \rightarrow 0
$$

An analogous calculation for the shot-noise provides

$$
S N_{-}=\left\langle I_{s}\right\rangle+\left\langle I_{i}\right\rangle \simeq \frac{g \Omega_{\mathrm{gvs}}}{\sin \epsilon} \quad \text { for } \epsilon \rightarrow 0
$$

Both quantities in principle diverge on approaching threshold, but at a finite distance from threshold their ratio gives

$$
\begin{aligned}
\frac{\mathcal{V}_{-}(\Omega)}{S N_{-}} & \simeq \frac{1}{2}\left[|\phi(\Omega)|^{2}\left|e^{i \beta(\Omega)}-g \operatorname{sinc}[\gamma(\Omega)]\right|^{2}+(\Omega \rightarrow-\Omega)\right] \quad \text { for } \epsilon \rightarrow 0 \\
& =\frac{1}{2}\left[\left|U_{s}(\Omega)-V_{i}^{*}(-\Omega) e^{i k_{s} l_{c}+i \phi_{p}}\right|^{2}+(\Omega \rightarrow-\Omega)\right]
\end{aligned}
$$

Remarkably, the last expression coincides with the quadrature squeezing spectra $\Sigma_{ \pm}(\Omega)$ in Eq. (33), evaluated at the fixed phase angles $\phi_{s}+\phi_{i}=k_{s} l_{c}+\phi_{p}=2 \theta(0)$, as confirmed by a comparison between the spectra in figures $4 \mathrm{p}$ and 7. Thus

$$
\frac{\mathcal{V}_{-}(\Omega)}{S N_{-}}=\Sigma_{ \pm}(\Omega) \approx \frac{\sqrt{g^{2}+\tilde{\Omega}^{2}}-g \sin \sqrt{g^{2}+\tilde{\Omega}^{2}}}{\sqrt{g^{2}+\tilde{\Omega}^{2}}+g \sin \sqrt{g^{2}+\tilde{\Omega}^{2}}} \quad \text { for } g \rightarrow \frac{\pi}{2}
$$

where $\tilde{\Omega}=\Omega / \Omega_{\mathrm{gvs}}$, and the last formula at r.h.s is obtained by using the linear approximation 11 and setting $\beta(\Omega) \simeq 0[10$. Notice however that, at difference with the quadrature spectra, in the region where squeezing is present (say $\Omega \leq 2.7 \Omega_{\mathrm{gvs}}$ ) the curve $\mathcal{V}_{-} / S N_{-}$remains practically identical passing from the highly stimulated regime $g \simeq \pi / 2$ to the spontaneous regime $g \ll 1$. In the latter case, for $g \rightarrow 0$, the function $|\phi(\Omega)|^{2}=1+\left|V_{s}(\Omega)\right|^{2} \rightarrow 1$, and can be substituted with unity in Eqs. (53) and (54) (this amounts to keeping only the leading order terms in $g^{2} \ll 1$ ). Then

$$
\frac{\mathcal{V}_{-}(\Omega)}{S N_{-}} \simeq 1-\operatorname{sinc}\left(\frac{\Omega}{\Omega_{\text {gvs }}}\right) \quad \text { for } g \rightarrow 0
$$

Finally, we investigate the noise in the difference of photon numbers collected over a finite time window $T_{d}$. Results are reported in Fig, which plots $\left\langle\left(\delta N_{-}\right)^{2}\right\rangle$ divided by the shot noise $\left\langle N_{s}\right\rangle+\left\langle N_{i}\right\rangle$ as a function of $T_{d} / \tau_{\mathrm{gvs}}$ for different values of the parametric gain.

In the spontaneous regime (limit $g \rightarrow 0$ ) it is possible to derive an approximated expression for this noise variance A):

$$
\frac{\left\langle\left(\delta \hat{N}_{-}\right)^{2}\right\rangle}{\left\langle\hat{N}_{s}\right\rangle+\left\langle\hat{N}_{i}\right\rangle} \simeq\left\{\begin{array}{ll}
1-\frac{T_{d}}{2 \tau_{\mathrm{gvs}}} & \text { for } T_{d} \leq \tau_{\mathrm{gvs}} \\
\frac{\tau_{\mathrm{gvs}}}{2 T_{d}} & \text { for } T_{d}>\tau_{\mathrm{gvs}}
\end{array} \quad \text { for } g \rightarrow 0\right.
$$

This function fits perfectly the curve obtained through numerical integration of Eq. (46) for small values of $g$ (open circles in Fig 8), and actually describes qualitatively the behaviour of $\frac{\left\langle\left(\delta \hat{N}_{-}\right)^{2}\right\rangle}{\left\langle\hat{N}_{s}\right\rangle+\left\langle\hat{N}_{i}\right\rangle}$ in all regimes, ranging from well below the MOPO threshold, to close to it. In particular, it shows that the noise in the photon number difference is reduced by $50 \%$ below shot-noise at $T_{d}=\tau_{\text {gvs }}$, and then approaches asymptotically zero.

In conclusion, the answer to our question is clear: in order to obtain sub-shot noise fluctuations in the difference of the photon numbers a collection time larger than $\tau_{\text {gvs }}$ is enough in any regime. This may appear perhaps surprising, because, according to the results presented in [9], close to threshold the cross correlation of twin beams $G_{s i}^{(2)}\left(t, t^{\prime}\right)=\left\langle\delta \hat{I}_{s}(t) \delta \hat{I}_{i}\left(t^{\prime}\right)\right\rangle$ acquires a slowly decaying exponential tail $\approx e^{-2 g \epsilon \frac{\left|t-t^{\prime}\right|}{\tau_{\text {gvs }}}}$, originating from stimulated PDC in combination with backpropagation [9]. However, according to the results presented here, the temporal correlation of the intensity difference remains confined to the smaller time $\tau_{\text {gvs }}$ characteristic of the spontaneous regime. Thus apparently the stimulated processes do not contribute to the correlation of the intensity difference. Mathematically,this happens because the same exponential tails appear in the autocorrelation functions $G_{j j}^{(2)}\left(t, t^{\prime}\right)[9]$ and cancel out the long-ranged part of the cross-correlation in the expression (47). Intuitively, in order to have a noise below the shotnoise of random processes, it is enough to collect in the two arms all the twin photons originating from the same primary down-conversion processes, whose delay cannot exceed their transit time $\tau_{\text {gvs }}$ across the slab. 


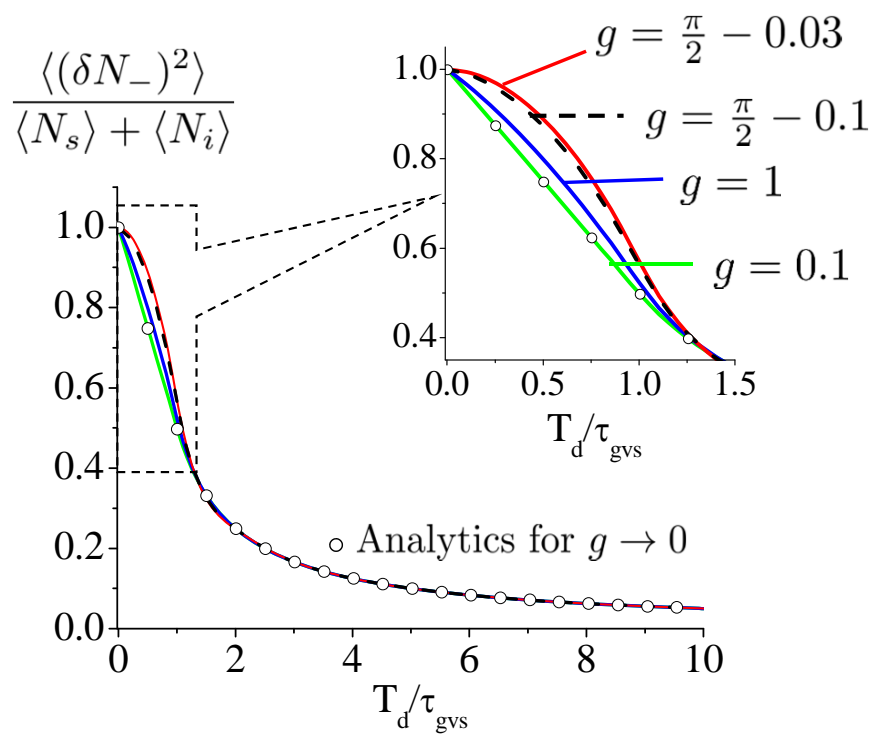

FIG. 8: Noise in $\hat{N}_{-}$normalized to the shot noise level $\left\langle N_{s}\right\rangle+\left\langle N_{i}\right\rangle$ as a function of the detection time $T_{d}$. The formula 63 derived in the limit $g \rightarrow 0$ (open circles) perfectly fits the $g=0.1$ result (green line), and reproduces rather well the curves in all regimes. Parameters as in Fig 3

\section{CONCLUSIONS}

The analysis performed in this work has outlined several appealing features of the countinuous variable entanglement of counter-propagating twin beams. Particularly appealing is the narrowband character of the emission( order few Ghz), the high-level of quadrature squeezing and the stability of the squeezing angle, which could make this monolithic source a viable alternative to the cavity OPO.

In contrast, the standard cavityless co-propagating configuration, as well known, is highly multimode and broadband (order tens of Thz). While such a spectrally multimode entanglement may represent an important resource for some applications, it is clear from our analysis that, especially in the high-gain, it would be hard to detect squeezing inside the whole bandwidth, because the squeezing-angle rotates rapidly with frequency, so that the contribution of the antisqueezed quadrature enters rapidly into play. Thus, contrary to what is usually thought(see e.g. [13]), it would be probably hard to detect and exploit squeezing over the entire huge PDC bandwidth. In contrast, the MOPO offers a high stability of the squeezing angle, which can be seen as a consequence of the peculiar time scale $\tau_{\text {gvs }}$ involved in the counterpropagating interaction, which is much longer than the time scales $\tau_{\text {gvm }}, \tau_{\text {gvd }}$ characterizing co-propagating light waves.

In the second part of the work we addressed the problem of the photon-number correlation in the MOPO. Perhaps surprisingly we have found that while the correlation time of twin beams has a critical divergence on approaching the threshold, the correlation of their intensity difference remains short ranged, and confined to the time $\tau_{\text {gvs }}$ characteristic of the spontaneous regime. This result, clearly positive for applications, means that sub-shot noise fluctuations of the photon-number difference can be measured within a finite detection time.

\section{Appendix A}

We derive here an expression for the noise of the photon-number difference $\hat{N}_{-}=\hat{N}_{s}-\hat{N}_{i}$, valid in the limit $g \rightarrow 0$ (spontaneous regime). We use the approximated expressions for the second-order field correlations derived in Ref. 9 ] in the same limit

$$
\begin{aligned}
\left\langle\hat{A}_{s}^{\text {¡out }}\left(t_{s}\right) \hat{A}_{s}^{\text {out }}\left(t_{s}^{\prime}\right)\right\rangle & \approx \frac{g^{2}}{2 \tau_{\mathrm{gvs}}} \operatorname{Triangle}\left(\frac{t_{s}-t_{s}^{\prime}}{2 \tau_{\mathrm{gvs}}}\right) \\
\left\langle\hat{A}_{s}^{\text {out }}\left(t_{s}\right) \hat{A}_{i}^{\text {out }}\left(t_{i}\right)\right\rangle & \approx \frac{g e^{i \phi_{p}+i k_{s} l_{c}}}{2 \tau_{\mathrm{gvs}}} \operatorname{Rect}\left(\frac{t_{s}-t_{i}}{2 \tau_{\mathrm{gvs}}}\right)
\end{aligned}
$$


where:

- Triangle $(x)=1-|x|$ if $|x|<1$, Triangle $(x)=0$ elsewhere is the triangular function,

- $\operatorname{Rect}(x)=1$ if $|x|<1 / 2, \operatorname{Rect}(x)=0$ elsewhere is the rectangular box function,

-we have omitted terms related to the slow phase $\beta(\Omega)$ which are on the order $\tau_{\mathrm{gvm}} / \tau_{\mathrm{gvs}}$.

Accordingly, the mean intensity is given by $\left\langle\hat{I}_{s}\right\rangle=\left\langle\hat{I}_{i}\right\rangle=\frac{g^{2}}{2 \tau_{g v s}}$. Then the shot-noise is $\left\langle\hat{N}_{s}\right\rangle+\left\langle\hat{N}_{i}\right\rangle=g^{2} \frac{T_{d}}{\tau_{g v s}}$. The intensity correlation functions defined by Eqs.449), (50) up to leading order in $g \ll 1$ become

$$
\begin{aligned}
& G_{j j}\left(t, t^{\prime}\right)=\delta\left(t-t^{\prime}\right)\left\langle I_{j}\right\rangle+O\left(g^{4}\right) \simeq \frac{g^{2}}{2 \tau_{\mathrm{gvs}}} \delta\left(t-t^{\prime}\right) \\
& G_{s i}\left(t, t^{\prime}\right) \simeq \frac{g^{2}}{4 \tau_{\mathrm{gvs}}^{2}} \operatorname{Rect}\left(\frac{t-t^{\prime}}{2 \tau_{\mathrm{gvs}}}\right)
\end{aligned}
$$

By inserting the approximated expressions $(\mathrm{A3})$ and $\mathrm{A} 4$ into the correlation of the intensity difference in Eq. (47), and evaluating the simple integrals involved in Eq. (45), we finally obtain

$$
\frac{\left\langle\left(\delta \hat{N}_{-}\right)^{2}\right\rangle}{\left\langle\hat{N}_{s}\right\rangle+\left\langle\hat{N}_{i}\right\rangle} \simeq\left\{\begin{array}{ll}
1-\frac{T_{d}}{2 \tau_{\mathrm{gvs}}} & \text { for } T_{d} \leq \tau_{\mathrm{gvs}} \\
\frac{\tau_{\mathrm{ggs}}}{2 T_{d}} & \text { for } T_{d}>\tau_{\mathrm{gvs}}
\end{array} \quad \text { for } g \rightarrow 0\right.
$$

[1] J. G. Rarity, P. R. Tapster, J. A. Levenson, J. C. Garreau, I. Abram, J. Mertz, T. Debuisschert, A. Heidmann, C. Fabre and E. Giacobino, Applied Physics B 55 (Sep 1992) 250.

[2] A. C. Busacca, C. L. Sones, V. Apostolopoulos, R. W. Eason and S. Mailis, Appl. Phys. Lett. 81 (2002) 4946.

[3] C. Canalias, V. Pasiskevicius, R. Clemens and F. Laurell, Appl. Phys. Lett. 82 (2003) 4233.

[4] S. E. Harris, Appl. Phys. Lett. 9 (1966) 114.

[5] C. Canalias and V. Pasiskevicius, Nat. Photon. 1 (Jun 2008) 459.

[6] A. Christ, A. Eckstein, P. J. Mosley and C. Silberhorn, Opt. Expr. 17 (Mar 2009) 3441.

[7] A. Gatti, T. Corti and E. Brambilla, Phys. Rev. A 92 (Nov 2015) p. 053809.

[8] A. Gatti and E. Brambilla, ArXiv e-prints (June 2017)

[9] T. Corti, E. Brambilla and A. Gatti, Phys. Rev. A 93 (Feb 2016) p. 023837.

[10] A. Gatti, T. Corti and E. Brambilla, Phys. Rev. A 96 (Jul 2017) p. 013820.

[11] M. Chekhova, G. Leuchs and M. ukowski, Optics Communications 337 (2015) 27 . Macroscopic quantumness: theory and applications in optical sciences.

[12] Y. Eto, T. Tajima, Y. Zhang and T. Hirano, Opt. Express 16 (Jul 2008) 10650.

[13] F. Kaiser, B. Fedrici, A. Zavatta, V. D'Auria and S. Tanzilli, Optica 3 (Apr 2016) 362.

[14] T. Suhara and M. Ohno, IEEE Journal of Quantum Electronics 46 (2010) 1739 .

[15] Y. Ding and J. Khurgin, Quantum Electronics, IEEE Journal of 32 (Sep 1996) 1574.

[16] D. Nikogosian, Nonlinear Optical Crystals: A Complete Survey (Springer, 2005).

[17] C. C. Gerry and P. L. Knight, Introductory Quantum Optics 2005.

[18] A. Gatti, R. Zambrini, M. San Miguel and L. Lugiato, PHYSICAL REVIEW A 68 (NOV 2003)

[19] M. D. Reid, Phys. Rev. A 40 (Jul 1989) 913.

[20] Z. Y. Ou, S. F. Pereira, H. J. Kimble and K. C. Peng, Phys. Rev. Lett. 68 (Jun 1992) 3663.

[21] E. Brambilla, A. Gatti, M. Bache and L. Lugiato, PHYSICAL REVIEW A 69 (FEB 2004)

[22] A. Gatti, E. Brambilla, M. Bache and L. Lugiato, PHYSICAL REVIEW LETTERS 93 (AUG 272004 )

[23] C. Gardiner, Quantum noiseSpringer series in synergetics, Springer series in synergetics (Springer-Verlag, 1991). 